\title{
A Level Line Selection Approach for Object Boundary Estimation
}

\author{
Charles Kervrann, Mark Hoebeke and Alain Trubuil \\ INRA - Biométrie \\ Domaine de Vilvert, 78352 Jouy-en-Josas, France \\ \{Charles.Kervrann,Mark.Hoebeke,Alain.Trubuil\}@jouy.inra.fr
}

\begin{abstract}
An energy model-based approach for estimating object boundaries is presented. We study a particular energy, which minimizer can be determined. The method estimates the unknown number of objects and draws object boundaries by selecting the "best" level lines computed from level sets of the original image. Unlike previous standard methods, the proposed method does not require iteration for minimizing the energy. In addition, our segmentation algorithm combines anisotropic diffusion-based regularization with level line selection to extract smooth object boundaries. Experimental results on $2 \mathrm{D}$ biomedical and meteorological images are reported.
\end{abstract}

\section{Introduction}

Image segmentation consists in partitioning the image into homogeneous regions ideally corresponding to physical objects. Several different methods have already been proposed in the literature: local filtering methods [8], region growing techniques [20] or global optimization techniques $[5,19,14,10]$. Many of them rely on the design and minimization of an energy function which captures the interaction between models and image data. They can be roughly classified into two main categories according to features. The first category of approaches relies on gradient features near object boundaries whereas the other one examines the features homogeneity inside object boundaries. Nevertheless, several methods combine both approaches [24].

Among the boundary-based approaches, filtering techniques (edge detectors [8]) and energy-based active contours (snakes [12] and variants) have been used to detect discontinuities or to extract continuous contour lines. Geodesic active contours $[13,7]$ based on the theory of surfaces evolution and geometric flows have been introduced to detect an arbitrary number of objects in the image. These models precisely delineate object boundaries but can rely on unreliable local information to make a decision.
In region-based approaches, the segmentation model aims at splitting or merging the image into zones the most homogeneous as possible. Homogeneity is traditionally measured by a given cost functional [17] or a Bayesian criterion $[18,10,24]$. In that case, the boundaries are the set of curves that minimizes a global energy function. Some energy models are based on a discrete model of the image, such as Markov random fields $([9,1,14,10]$ whereas variational models are based on a continuous model of the image $[5,19,18]$. Specific a priori constraints generally encourage the emergence of few regions which boundaries are regular [19, 14]. High-level constraints [10] can be introduced to detect specific complex objects. All these methods are generally robust to noise but computationally demanding if stochastic iterative procedures are used to conduct the minimization $[5,14,10]$. This motivates the search for energy models allowing non-iterative inference.

The aim of this paper is to provide energy models, which minimizers can be determined in advance. Accordingly, energy minimization methods and iterative algorithms are not necessary to solve the optimization problem. We have modified a particular segmentation energy first introduced in a discrete setting by Beaulieu and Goldberg [2]. The original model tends to obtain a partition with a few number of regions and small variances without a priori knowledge on the image. However, the energy which is efficiently minimized using a split-and-merge algorithm [2], has the drawback of giving no control on the smoothness of the object boundaries [17]. Here, our approach is completely different to determine its minimizer. Indeed, we prove in Section 3 that the set of curves that minimizes the modified energy is a family of level lines defined from level sets of the image. In that case, the method is deterministic and equivalent to a procedure that selects the "best" level lines delimiting object boundaries. Additionally, we combine anisotropic diffusion-based regularization with level line selection to estimate smooth object boundaries. For the completeness of the paper, a description of this segmentation algorithm is included in section 4. In section 5, experiments on several examples demonstrate the effectiveness of the approach. 


\section{Preliminaries}

Our theoretical setting is the following. One assumes the existence of an unknown function $f$, i.e. the true image, from our image domain $S:[0, a] \times[0, b]$ to $\mathbb{R}$. The minimization of the energy on $f$ defines the true segmentation. One observes a regular discretization of a corrupted image $y=f+\varepsilon$, where $\varepsilon$ is a gaussian white noise (variance $\sigma_{\varepsilon}^{2}$ ). The observations are therefore

$$
y(s)=f(s)+\varepsilon(s), \quad 0 \leq s \leq N
$$

with $s=(x, y) \in S$ is on one of $N$ pixels. A nonparametric estimator of $f$ is built and plugged in the defined energy. We assume that the minimization with respect to $f$ leads to an image partition into regions.

Let $\Omega_{i} \subset S, i=1, \ldots, P$ an non-empty image domain or object. We associate with the unknown domains $\Omega_{i}$ the following energy, inspired from $[2,11]$ :

$$
\begin{aligned}
& E\left(f, \Omega_{1}, \ldots, \Omega_{P}\right)= \\
& \quad \sum_{i=1}^{P} \int_{\Omega_{i}}\left(f(x, y)-\overline{f_{\Omega_{i}}}\right)^{2} d x d y+\lambda J\left(P, \Omega_{i}\right)
\end{aligned}
$$

where $\overline{f_{\Omega_{i}}}$ denotes the maximum likelihood estimate of the mean of $f$ over $\Omega_{i}$

$$
\overline{f_{\Omega_{i}}}=\frac{1}{\left|\Omega_{i}\right|} \int_{\Omega_{i}} f(x, y) d x d y, \quad\left|\Omega_{i}\right|=\int_{\Omega_{i}} d x d y
$$

$\lambda$ is a given positive weight and $J\left(P, \Omega_{i}\right)$ is a a priori functional corresponding to a loose constraint to be defined. Note that (2) can be interpreted as a maximum a posteriori estimator when we assume all sites are independent and all sites belonging to a given object $\Omega_{i}$ have identical distributions characterized by parameters $\left\{\overline{f_{\Omega_{i}}}, \sigma_{\varepsilon}^{2}\right\}$.

Our aim is now to define objects in $f$. Therefore, we define the following class $C_{P}, P \geq 1$ of admissible objects

$$
\begin{aligned}
C_{P}=\{ & \left(\Omega_{1}, \ldots, \Omega_{P}\right) \subset S \text { are regular, closed and connected; } \\
& 1 \leq i, j \leq P-1, i \neq j \Longrightarrow \Omega_{i} \cap \Omega_{j}=\emptyset ; \\
& \left.\Omega_{i} \cap \Omega_{P}=\emptyset ; \quad \cup_{i=1}^{P} \Omega_{i}=S\right\}
\end{aligned}
$$

the subsets $\left(\Omega_{1}, \ldots, \Omega_{P-1}\right)$ are the objects of the image and $\Omega_{P}$ is the background. When $P=1$, no object is on the image. An optimal segmentation of image $f$ over $C_{P}$ is by definition a global minimum of the energy (when exists)

$$
\begin{aligned}
& \left(\Omega_{1}^{\star}, \ldots, \Omega_{P^{\star}}^{\star}\right)= \\
& \operatorname{argmin}_{P \geq 1} \operatorname{argmin}_{\left(\Omega_{1}, \ldots, \Omega_{P}\right) \in C_{P}} E\left(f, \Omega_{1}, \ldots, \Omega_{P}\right) .
\end{aligned}
$$

A direct minimization with respect to all unknown domains $\Omega_{i}$ and parameters $\overline{f_{\Omega_{i}}}$ is a very intricate problem. In the next section, we prove that the object boundaries are level lines of the nonparametric estimator of $f$ if we choose $J\left(P, \Omega_{i}\right)=P$ or $J\left(P, \Omega_{i}\right)=\sum_{i=1}^{P-1}\left|\Omega_{i}\right|$. In that case, $J\left(P, \Omega_{i}\right)$ gives no control on the smoothness of boundaries and $\lambda$ can be interpreted as a scale parameter that only tunes the number of regions $[2,17]$.

\section{Estimator}

Our plug-in estimator is defined by (when exists)

$\left(\widehat{\Omega}_{1}, \ldots, \widehat{\Omega}_{\widehat{P}}\right)=$

$\operatorname{argmin}_{P \geq 1} \operatorname{argmin}_{\left(\Omega_{1}, \ldots, \Omega_{P}\right) \in C_{P}} E\left(\widehat{f}, \Omega_{1}, \ldots, \Omega_{P}\right)$.

The question of the existence of an admissible global minimum for energies like Beaulieu and Goldberg's energy [2] or Mumford and Shah's energy [19] is a difficult problem (see [17] for more details). Here, our aim is not to investigate conditions for having an admissible global minimum.

\subsection{Minimizer description}

In what follows, we make an ad-hoc assumption ensuring the existence of an unique minimum of the energy taken for functions in an neighborhood of the true image $f$ [11]. In that case, we propose the following lemma

Lemma If there exists an unique admissible global minimum for each function of a small neighborhood of $f$ and that no pathological minimum exists [11], then

$$
\widehat{f}_{\mid \partial \Omega_{i}} \equiv \mu_{i}, \quad i=1, \ldots, \widehat{P}-1 .
$$

i.e. the border of each $\Omega_{i}$ is a boundary of a level set of $\widehat{f}$.

Proof of lemma Without loss of generality, we prove this lemma for one object $\Omega$ and a background $\Omega^{c}$, where $\Omega^{c}$ denotes the closure of the complementary set of $\Omega$. For two sets $A$ and $B$, denote $\int_{A-B} \widehat{f} \equiv \int_{A} \widehat{f}-\int_{B} \widehat{f}$. Let $\Omega_{\delta}$ be a small perturbation of $\Omega$, i.e. the Hausdorff distance $d_{\infty}\left(\Omega_{\delta}, \Omega\right) \leq \delta$. Then, we define

$$
\begin{gathered}
\int_{\Omega_{\delta}-\Omega} \mathbb{I I} \triangleq\left|\Omega_{\delta}\right|-|\Omega| \\
\left(\int_{\Omega_{\delta}} \widehat{f}\right)^{2}-\left(\int_{\Omega} \widehat{f}\right)^{2}=2 \int_{\Omega} \widehat{f} \int_{\Omega_{\delta}-\Omega} \widehat{f}+\left(\int_{\Omega_{\delta}-\Omega} \widehat{f}\right)^{2} .
\end{gathered}
$$

The difference between the involved energies is equal to $E\left(f, \Omega_{\delta}, \Omega_{\delta}^{c}\right)-E\left(f, \Omega, \Omega^{c}\right)=T_{1}+T_{2}+T_{3}+T_{4}$, with

$$
\begin{aligned}
& T_{1}=\int_{\Omega_{\delta}} \hat{f}^{2}-\int_{\Omega} \hat{f}^{2}, \quad T_{2}=-\frac{1}{\left|\Omega_{\delta}\right|}\left(\int_{\Omega_{\delta}} \hat{f}\right)^{2}+\frac{1}{|\Omega|}\left(\int_{\Omega} \hat{f}\right)^{2} \\
& T_{3}=\int_{S-\Omega_{\delta}} \hat{f}^{2}-\int_{S-\Omega} \hat{f}^{2}
\end{aligned}
$$




$$
T_{4}=-\frac{1}{1-\left|\Omega_{\delta}\right|}\left(\int_{S-\Omega_{\delta}} \hat{f}\right)^{2}+\frac{1}{1-|\Omega|}\left(\int_{S-\Omega} \hat{f}\right)^{2} .
$$

Using (6)

$$
\begin{aligned}
T_{1}= & \int_{\Omega_{\delta}-\Omega} \hat{f}^{2} \\
T_{2}= & -\frac{2}{|\Omega|} \int_{\Omega_{\delta}-\Omega} \hat{f} \int_{\Omega} \hat{f}-\frac{1}{|\Omega|}\left(\int_{\Omega_{\delta}-\Omega} \hat{f}\right)^{2} \\
& +\frac{1}{|\Omega|^{2}} \int_{\Omega_{\delta}-\Omega} \mathbb{I}\left(\int_{\Omega} \hat{f}\right)^{2}+O\left(\int_{\Omega_{\delta} \Delta \Omega} \mathbb{I}\right)^{2}
\end{aligned}
$$

and similar expression for $T_{3}$ and $T_{4}$. Define

$$
\begin{gathered}
m_{0}=\int_{\Omega} \mathbb{I I}, \quad m_{1}=\int_{\Omega} \widehat{f}, \quad K_{1}=\int_{S} \widehat{f} \\
P_{0}=-\frac{2 m_{1}}{m_{0}}+\frac{2\left(K_{1}-m_{1}\right)}{1-m_{0}}, \quad P_{1}=\frac{m_{1}^{2}}{m_{0}^{2}}-\frac{\left(K_{1}-m_{1}\right)^{2}}{\left(1-m_{0}\right)^{2}} .
\end{gathered}
$$

Let $M$ be a fixed point of the border $\partial \Omega$. Choose $\Omega_{\delta}$ such that $\partial \Omega_{\delta}=\partial \Omega$ except on a small neighborhood of $M$. The energy having a minimum for $\Omega, \widehat{f}(M)$ needs to be solution of the following equation

$$
P_{0} \widehat{f}(M)+P_{1}=0 \text {. }
$$

Equation (10) has an unique solution. $P_{0}$ and $P_{1}$ do not depend on $M$ and $\widehat{f}(M)$ and $P_{0} \neq 0$. $\widehat{f}$ is continuous and $\partial \Omega$ is a connected curve. Therefore $\widehat{f}(M)$ is constant when $M$ covers $\partial \Omega$.

In conclusion, we proved that the minimizer is a family of iso-intensity curves of the image. They can be determined by boundaries of level sets defined in Section 3.2.

\subsection{Image representation by level sets}

As argued by the Mathematical Morphology [15, 23], it follows that the basic information of an image is contained in the family of its binary shadows or level sets, that is, in the family of sets $\mathcal{S}_{\eta}$ defined by

$$
\mathcal{S}_{\eta}=\{s \in S: f(s) \geq \eta\}
$$

for all values of $\eta$ in the range of $f$. Level sets provide a complete, contrast invariant image representation unlike representation by "edges" or "luminance" [16].

A recent variant of this representation is proposed in [6] by considering the boundary of level sets, that is the level lines. This representation does not differ with respect to the set of level sets. Level sets can be computed from all possible connected components which are based both on the image gray levels and spatial relations between pixels. To extract a connected component of a level set $\mathcal{S}_{\eta}$, we threshold the image at the gray level $\eta$ and extract the components of the binary image we obtain. In the following, we basically consider that the level lines are built from connected components $\Omega_{i}$ extracted at each level set. They are just a set of $\eta$-isovalue pixels at the borders $\partial \Omega_{i}$ of connected components. More precise concepts about level lines, level sets and connected components can be found in [16]. Nevertheless level lines, i.e. object boundaries, are not generally regular curves in the image. Therefore, anisotropic diffusion is introduced to remove noise and fine-scale details and preserve well-localized smooth level lines.

\subsection{Regularization of level lines}

Anisotropic diffusion aims at smoothing original image while preserving brightness discontinuities [21]. Black et al. showed that anisotropic diffusion can be interpreted as a robust procedure that estimates a piecewise constant image from noisy input image [4]. They derived a relationship between anisotropic diffusion and the error norm and influence function in the robust estimation framework, yielding to new anisotropic diffusion equations.

In [4] it is established that the Tukey's biweight robust error norm produces sharper discontinuities than the original "stopping-edge" function introduced by Perona and Malik [21]. The corresponding anisotropic diffusion equations are

$$
\begin{aligned}
& f_{t+1}(s)=f_{t}(s)+\frac{\tau}{\left|\mathcal{G}_{s}\right|} \sum_{p \in \mathcal{G}_{s}} z_{s p}\left(f_{t}(p)-f_{t}(s)\right) \\
& z_{s p}= \begin{cases}{\left[1-\left(\left(f_{t}(p)-f_{t}(s)\right) / \sigma\right)^{2}\right]^{2}} & \left|f_{t}(p)-f_{t}(s)\right| \leq \sigma \\
0 & \text { otherwise }\end{cases}
\end{aligned}
$$

where $f_{t}(s)$ is a filtered image of $f(s), t$ denotes iterations, $\tau$ is a strictly positive constant that determines the rate of diffusion, $\mathcal{G}_{s}$ represents the first-order spatial neighborhood of $s,\left|\mathcal{G}_{s}\right|$ is the number of neighbors, $\sigma$ is a "robust scale" parameter [3] and $z_{s p} \in[0,1]$ is analog to a line process. The line process indicates the presence ( $z$ close to 0 ) or absence ( $z$ close to 1 ) of discontinuities or outliers.

Scale parameter $\sigma$ can be estimated in a robust way (see [22]). Here, we adaptively estimate a scale parameter $\sigma_{i}=$ $k \tilde{\sigma}_{i}$ for each object $\Omega_{i}$ where $k$ is a positive constant,

$$
\tilde{\sigma}_{i}=2,6477 \sqrt{\frac{2}{\left|\Omega_{i}\right|} \sum_{l=0}^{\left|\Omega_{i}\right| / 2} r_{O(l)}^{2}}
$$

and $r_{O(1)}^{2} \leq r_{O(2)}^{2} \leq \cdots \leq r_{O\left(\left|\Omega_{i}\right| / 2\right)}^{2}$ are the $\left|\Omega_{i}\right| / 2$ ordered squared residuals. The constant factor makes $\tilde{\sigma}_{i}$ roughly unbiased at gaussian error distributions [22]. Only the first ranked half of data belonging to $\Omega_{i}$ is used to estimate $\sigma_{i}$ and $r_{O(\cdot)}^{2}$ is computed from the image gradient magnitude approximation (in a particular direction) as $f_{t}(p)-f_{t}(s), p \in \mathcal{G}_{s}$. This filter creates consistent results with the level line selection approach. 
The connection between anisotropic diffusion and extraction of objects $\Omega_{i}$ is then established. At each iteration $t$, data are smoothed in each region $\Omega_{i}$ using (12) where $\sigma_{i}$ is substituted to $\sigma$. In that case, the number of iterations to converge can be easily controlled. The same level lines are invariably selected if the restored image is a piecewise smooth image. Usually this can be accomplished with less than 10 iterations in sufficient precision. Note that the two tasks would be independent in the original formulation in [21], for which the diffusion stopping rule is not well defined. This relationship is more underlined through a practical segmentation algorithm described in the next section.

\section{Numerical implementation}

To implement our level set image segmentation, a four step method is used. Given a smoothed image $f_{t}$ obtained using (12), the first step completes a mapping of each image pixel on a given level set. It is followed by an object extraction stage in which sets of simply connected pixels are extracted from the level sets. The connected components are then combined during the third step to form object configurations. Objects $\Omega_{i}$ are defined by connected components and $\partial \Omega_{i}$ are boundaries of connected components. In the final stage, calculations are performed on all object combinations. The configuration with the lowest energy is then selected as the best segmentation.

LEVEL SET CONSTRUCTION Let $f_{t}(s) \in\left[f_{\text {min }}, f_{\text {max }}\right]$ the image partitioned in $K=4,8$ or 16 equal-sized and non-overlapping intervals $\left\{\left[l_{1}, h_{1}\left[, \ldots,\left[l_{K}, h_{K}\right]\right\}\right.\right.$. A pixel $s$ will then belong to the interval $\left[l_{j}, h_{j}\left[\right.\right.$ if $l_{j} \leq f_{t}(s)<h_{j}$.

OBJECT EXTRACTION A crude way to build pixels sets corresponding to objects is to proceed to a connected components labeling and to associate each label with an object $\Omega_{i}$. The background $\Omega_{P}$ corresponds to the closure of the complementary set of objects $\Omega_{i}$. This amounts to building $K$ images $g_{t}^{j}, j=1, \ldots, K$ in which $g_{t}^{j}(s)=\eta, \forall s \in \mathcal{S}_{\eta}$ and $g_{t}^{j}(s)=0, \forall s \notin \mathcal{S}_{\eta}$. The list of connected components of each of these then forms the list of objects $\left\{\Omega_{1}, \ldots \Omega_{P-1}\right\}$.

CONFIGURATION DETERMINATION Having the final object list, configurations are combinations of objects of the $C_{p}$ class. These configurations can be built by enumeration of all possible object combinations, i.e. $2^{P-1}$ configurations. Nevertheless, we decide to discard objects which areas are under a predetermined threshold. Each possible configuration can then be represented by a binary number $b_{i}$ which is the binary expansion of $i\left(0 \leq i \leq 2^{P-1}-1\right)$. The value of each bit in $b_{i}$ determines the presence or absence of a given object in the configuration.
ENERGY COMPUTATION Each configuration represents a set of objects which in turn is a set of pixels. Energy calculations take the image intensities at each of these pixels to establish mean (3) and approximation error (2). Note that only energies corresponding to each object are computed once and stored, and energies corresponding to the background are efficiently updated for each configuration. The configuration that minimizes the global energy corresponds to the optimal segmentation. The time necessary to perform image segmentation essentially depends on the length of the object list, i.e. the number of connected components $P$. Nevertheless, all configurations are independent and could be potentially evaluated on suitable parallel architectures.

The steps of the algorithm are the following:

- Initialization: Let $f_{t_{0}}$ the original image data. Set parameters $K, \lambda, \tau$ and $k$.

- WhILe $f_{t+1}(s) \neq f_{t}(s), \forall s \in S$ REPEAT

- Image segmentation:

1. Level set construction

2. Object extraction

3. $2^{P-1}$ configurations determination

4. Energy computation and configuration selection with the lowest energy.

- Anisotropic diffusion: see (12).

- Updating of $\sigma_{i}$ and $k \leftarrow 0.9 k$ and increment $t$;

END WHILE.

\section{Experimental results}

We demonstrate segmentation results on synthetic images as well as on 2D confocal microscopy, medical and meteorological images. The algorithm parameters were set as follows: $K=8, J\left(P, \Omega_{i}\right)=\sum_{i=1}^{P-1}\left|\Omega_{i}\right|$ and regions which areas $\left|\Omega_{i}\right|<0.01 \times N$ are discarded, $\tau=1$ and the value of $k$ is initially set to 5 and linearly lowered by a factor of 0.9 at each iteration. The segmentation was terminated if the convergence criterion was met. Most segmentations required less than 10 iterations for convergence, according to image contents and noise, and took approximately $5 \mathrm{~s}$ per iteration on a $296 \mathrm{MHz}$ workstation.

To see the effect of adaptive estimation of $\sigma_{i}$ on segmentation results consider the simple example in Figure 1. The corrupted version with a white gaussian noise (s.d. 10) of a $256 \times 256$ image coming from the GdR ISIS is shown on the left. This image is composed of six objected against a background. The next column shows the segmentation results when $\sigma_{i}=\sigma, \forall \Omega_{i}$ and $\sigma$ is computed in a robust way for 

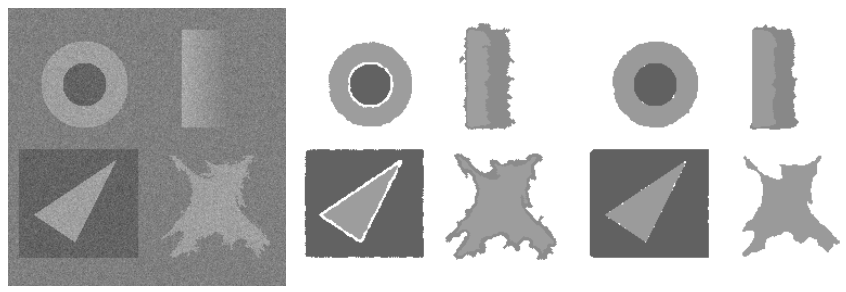

Figure 1. Segmentation results $(\lambda=650)$ after 5 iterations. Left: corrupted image. Middle: segmentation with $\sigma_{i}=\sigma, \forall \Omega_{i}$. Right: segmentation with $\sigma_{i} \neq \sigma_{j}, \forall\left(\Omega_{i}, \Omega_{j}\right)$.

the whole image. Background is labeled in "white" and undesirable regions are visible close to edges. The column on the right of Fig. 1 shows better results when $\sigma_{i}$ is adaptively computed for each $\Omega_{i}$ using (13). This modeling prevents oversmoothing of edges.

We have tested the proposed algorithm on 2D confocal microscopy images (Figs. 2-3), courtesy of INSERM 413 IFRMP $n^{\circ} 23$ (Rouen, France). Confocal fluorescence microscopy is a non-invasive technology for visualizing specimens in their natural tissue context. A 3D representation of the fluorescence-stained specimen and unstained background is built up from a stack of 2D image slices, referred to as optical sections. The first $115 \times 512$ image depicts a neurite in cultured cerebellar granule cells. This study aims at estimating calcium concentration in neurites. Figure 2 shows regions corresponding to three different calcium densities. The segment boundaries are correctly located and smooth. The second $256 \times 239$ confocal image depicts motility of two splitting neural cells. In this study, real-time changes in the shape of granule cells are examined. Figure 3 shows the detection of two objects of interest made up connected regions.

Figures 4-5 illustrate how our method selects the number of segments in a $2 \mathrm{D}$ medical MR image $(256 \times 228)$. in this application, background is previously eliminated by thresholding. The aim is to segment the corpus callosum (Fig. 4) or both the corpus callosum and brain (Fig. 5) by increasing $\lambda$. Semantic segmentations are obtained.

An example of cloud detection is provided in Fig. 6. The algorithm labeled seas and small clouds as "background". The significant clouds and continents are crudely extracted from the $383 \times 260$ image. Here, our approach can potentially provide an insight on the analyzed weather situation.

\section{Conclusion and perspectives}

In this paper, we have presented a level line selection approach for extracting structures in images. We proved that the minimizer of our segmentation energy can be directly determined. We introduced a robust anisotropic diffusion

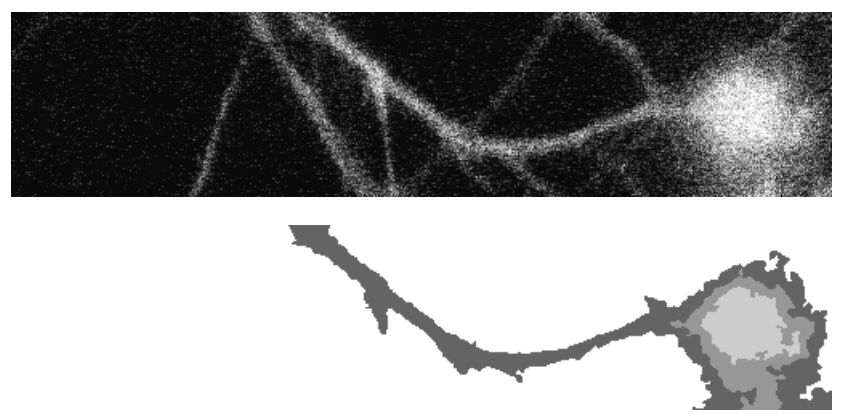

Figure 2. Segmentation in 2D confocal microscopy after $61 s$ and 10 iterations $(\lambda=650)$.
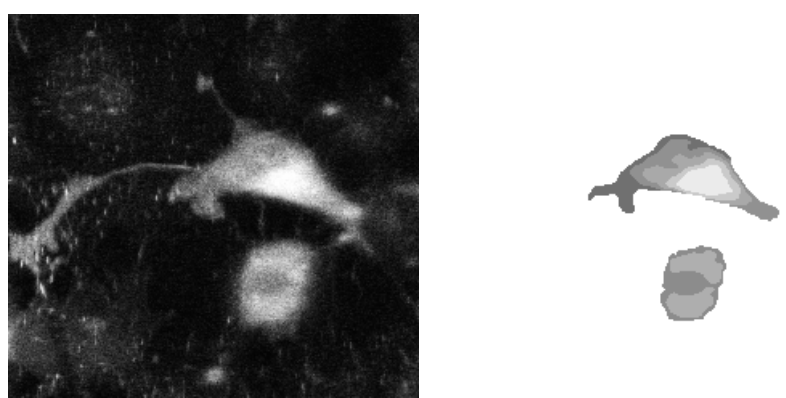

Figure 3. Segmentation in 2D confocal microscopy after $51 s$ and 8 iterations $(\lambda=3250)$.

framework to preserve sharper boundaries of objects and regularize level lines in the image. A total CPU time of a few seconds for segmenting a $256 \times 256$ image on a workstation makes the method attractive for many time-critical applications. The contribution of this approach has been illustrated on synthetic as well as real-world images. Several promising directions may be explored for continued research. First, we plan to examine new energy functions which minimizers can be determined as well. An other direction for future work is to extend the proposed approach to operate on multi-spectral 3D images.

\section{References}

[1] R. Azencott. Image analysis and markov fields. In Proc. of ICIAM SIAM Congress, Paris, France, 1987.

[2] J. Beaulieu and M. Goldberg. Hierarchy in picture segmentation: a stepwise optimization approach. IEEE Trans. Pattern Anal. and Machine Intell., 11(2):150-163, 1989.

[3] M. Black and A. Rangarajan. On the unification of line processes, outlier rejection, and robust statistics with applications in early vision. Int. J. Computer Vision, 19(1):57-92, 1996.

[4] M. Black, G. Sapiro, D. Marimont, and D. Heeger. Robust anisotropic diffusion. IEEE Trans. Image Processing, 7(3):421-432, 1998. 


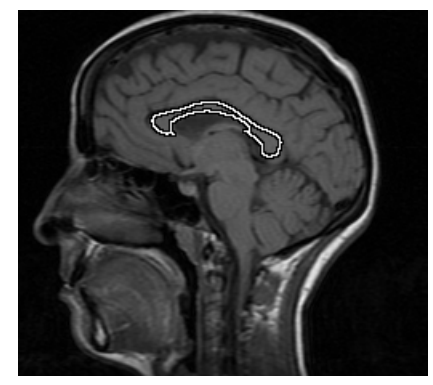

Figure 4. MR image segmentation after $4 s$ and only one iteration $(\lambda=32500)$.
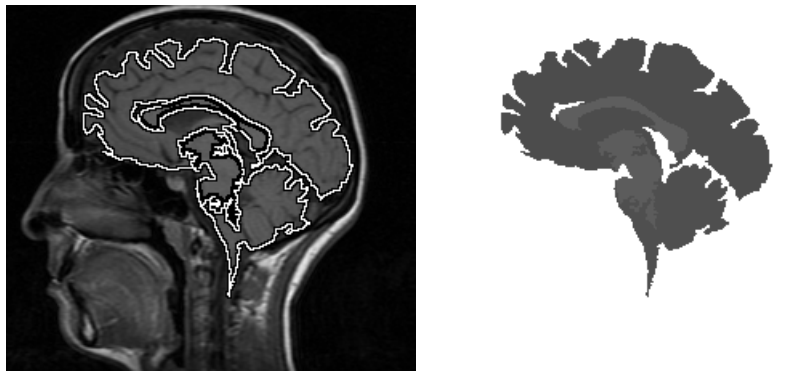

Figure 5. MR image segmentation after $4 s$ and only one iteration $(\lambda=6500)$.

[5] A. Blake and A. Zisserman. Visual reconstruction. MIT Press, Cambridge, Mass, 1987.

[6] V. Caselles, B. Coll, and J. Morel. Topographic maps. preprint CEREMADE, 1997.

[7] V. Caselles, R. Kimmel, and G. Sapiro. Geodesic active contours. Int J. Computer Vision, 22(1):61-79, 1997.

[8] R. Deriche. Using canny's criteria to derive a recursively implemented optimal edge detector. Int J. Computer Vision, 2(1):167-187, 1987.

[9] S. Geman and D. Geman. Stochastic relaxation, gibbs distributions, and the bayesian restoration of images. IEEE Trans. Pattern Anal. and Machine Intell., 6(6):721-741, 1984.

[10] U. Grenander and M. Miller. Representations of knowledge in complex systems. J. Royal Statistical Society, series B, 56(4):549-603, April 1994.

[11] J. Istas. Statistics of processes and signal-image segmentation. University of Paris VII, 1997.

[12] M. Kass, A. Witkin, and D. Terzopoulos. Snakes: active contour models. Int J. Computer Vision, 12(1):321-331, 1987.

[13] S. Kichenesamy, A. Kumar, P. Olver, and A. Yezzi. Conformal curvature flows: from transition to active contours. Archive for Rational Mechanics and Anal., 134:275-301, 1996.

[14] Y. Leclerc. Constructing simple stable descriptions for image partitioning. Int J. Computer Vision, 3:73-102, 1989.

[15] G. Matheron. Random sets and integral morphology. John Wiley, New York, 1975.

[16] P. Monasse and F. Guichard. Fast computation of a contrastinvariant image representation. IEEE Trans. Image Processing, 1999.
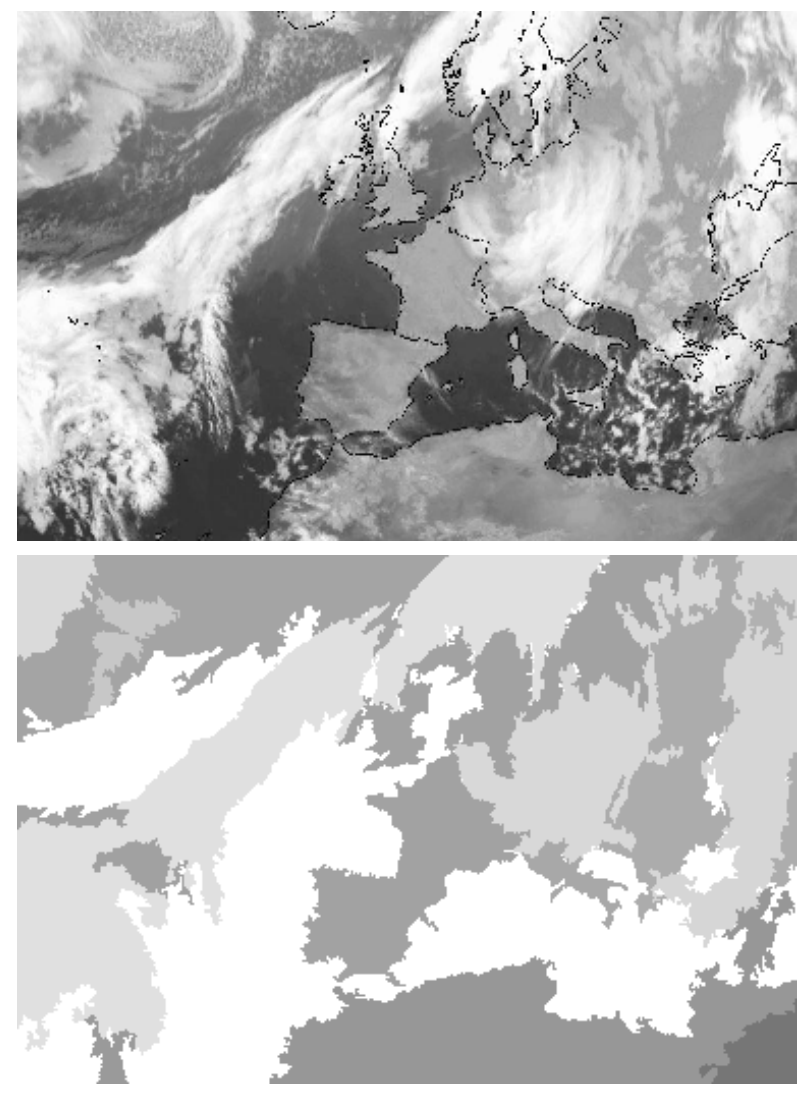

Figure 6. meteorological image segmentation after $18 s$ and only one iteration $(\lambda=2000)$.

[17] J. Morel and S. Solimini. Variational methods in image segmentation. Birkhauser, Boston, 1994.

[18] D. Mumford. The bayesian rationale for energy functionals. Geometry-driven diffusion in computer vision, pages 141153, Bart Romeny ed., Kluwer Academic, 1994.

[19] D. Mumford and J. Shah. Optimal approximations by piecewise smooth functions and variational problems. Сотmunication on Pure and applied Mathematics, 42(5):577-685, 1989.

[20] T. Pavlidis and Y. Liow. Integrating region growing and edge detection. IEEE Trans. Pattern Anal. and Machine Intell., 12:225-233, 1990.

[21] P. Perona and J. Malik. Scale-space and edge detection using anisotropic diffusion. IEEE Trans. Pattern Anal. and Machine Intell., 12(7):629-639, 1990.

[22] P. Rousseeuw and A. Leroy. Robust regression and outlier detection. Wiley Series in Probability and Mathematical Statistics, Wiley, 1987.

[23] J. Serra. Image Anal. and mathematical morphology. Academic Press, 1982.

[24] S. Zhu and A. Yuille. Region competition: unifying snakes, region growing, and bayes/mdl for multiband image segmentation. IEEE Trans. Pattern Anal. and Machine Intell., 18(9):884-900, 1996. 